\title{
Canadian
} Science Publishing

Canadian Journal of Physics

Revue canadienne de physique

\section{The Belgian Repository of Fundamental Atomic Data and Stellar Spectra}

\begin{tabular}{|c|c|}
\hline Journal: & Canadian Journal of Physics \\
\hline Manuscript ID & cjp-2016-0750.R1 \\
\hline Manuscript Type: & Article \\
\hline Date Submitted by the Author: & 16-Dec-2016 \\
\hline Complete List of Authors: & $\begin{array}{l}\text { Lobel, Alex; Observatoire Royale de Belgique, } \\
\text { Royer, Pierre; Katholieke Universiteit Leuven - Campus Arenberg, Instituur } \\
\text { voor Sterrenkunde } \\
\text { Martayan, Christophe; European Southern Observatory } \\
\text { Laverick, Michael; Katholieke Universiteit Leuven - Campus Arenberg, } \\
\text { Instituut voor Sterrenkunde } \\
\text { Merle, Thibault; Universite Libre Bruxelles Faculte des Sciences } \\
\text { David, Marc; Universiteit Antwerpen Faculteit Wetenschappen } \\
\text { Hensberge, Herman; Observatoire Royale de Belgique } \\
\text { Thienpont, Emmanuel; Vereniging Voor Sterrenkunde }\end{array}$ \\
\hline Keyword: & $\begin{array}{l}\text { stellar spectroscopy, spectral lines, quantitative spectroscopy, atomic line } \\
\text { data, atomic databases }\end{array}$ \\
\hline
\end{tabular}




\title{
The Belgian Repository of Fundamental Atomic Data and Stellar Spectra
}

\author{
A. Lobel ${ }^{1}$, P. Royer, C. Martayan, M. Laverick, T. Merle, M. David, H. Hensberge, and E. \\ Thienpont
}

Abstract BRASS is an international networking project of the Federal Government of Belgium for the development of a new public database providing accurate fundamental atomic data of vital importance for stellar spectroscopic research. The BRASS database will offer atomic line data we thoroughly test by comparing theoretical and observed stellar spectra. We are in the course of performing extensive quality assessments of selected atomic input data with advanced radiative transfer spectrum synthesis calculations that we compare in detail to high-resolution Mercator-HERMES and ESO-VLT-UVES spectra of very high signal-to-noise ratios for about 30 hot and cool bright stars of B, A, F, G, and K spectral types.

The new database will provide the tested and validated values of absorption lines we retrieve from various existing atomic repositories such as NIST and VAMDC. The validated atomic datasets, combined with the observed and theoretical spectra, will be interactively offered online at brass.sdf.org. The combination of these datasets is a novel approach for its development, which will provide a universal reference for advanced stellar spectroscopic research.

We present the atmospheric parameter results of a subset of 5 benchmark stars observed with signal-to noise ratios of 800-1200. The observed and theoretical spectra of the Sun and 51 Peg between $4000 \AA$ and $6800 \AA$ are offered online in the BRASS Data Interface. It also incorporates a new list of $\sim 900$ metal lines for which we compute blending below $5 \%$ of the equivalent width useful for detailed line profile modeling and synthetic spectrum fit quality assessments of atomic line data.

\section{Introduction}

Accurate oscillator strength values and line rest wavelengths are of central importance for the analysis of astrophysical spectra. Radiative transfer modeling of the detailed profiles of absorption lines in stellar spectra requires accurate atomic input data for investigating physical properties in the line formation region of stellar atmospheres. In recent years a number of online databases have become available offering impor-

A. Lobel ${ }^{1}$, P. Royer, C. Martayan, M. Laverick, T. Merle, M. David, H. Hensberge, and E. Thienpont. ${ }^{1}$ Royal Observatory of Belgium, Ringlaan 3, B-1180, Brussels, Belgium; University of Leuven, Belgium; European Southern Observatory, Paranal, Chile; Université Libre de Bruxelles, Belgium; University of Antwerp, Belgium; Royal Observatory of Belgium; Vereniging voor Sterrenkunde, Belgium

${ }^{1}$ Corresponding author (e-mail: Alex.Lobel@oma.be; alobel@sdf.org). tant atomic and molecular line data compiled from a large variety of sources in the scientific literature, such as VAMDC [1], NIST [2], ExoMol [3]. The quality of detailed theoretical modeling of stellar spectra strongly depends on the accuracy of the atomic data published in the literature and collected in these repositories. However, the accuracy of the provided atomic data is often not mentioned or simply unknown. It severely complicates the physical interpretation of the spectra for determining reliable atmospheric conditions and chemical composition in stellar population synthesis studies of large spectroscopic surveys. Quality tested atomic input data are for example urgently needed for compiling large homogeneous spectral libraries for processing and analyzing (i.e., with automatic spectral classification methods) over one hundred million ESA-Gaia spectra observed for all stellar spectral types [4].

The importance of fundamental atomic data in astrophysics and stellar physics cannot be overstated. It is the subject of periodic international conferences (such as ICAMDATA 19972016), science symposia (i.e., Uncertainties in atomic data and how they propagate in chemical abundances, Spain 2010: www.iac.es/congreso/atom), and joint workshops (i.e., Stellar atmospheres in the Gaia era: quantitative spectroscopy and comparative spectrum modelling, Brussels 2011: [5]). Multiple authors frequently insist on the issues with inaccurate fundamental atomic input data, and the lack of reference stars for validating the analysis methods over large stellar parameter spaces [e.g., 6, 7]. State-of-the-art research in this area relies on very few benchmark spectra, and is always limited in scope, for example [8] dispose of only 4 benchmark stars, all of solar type.

The BRASS project takes a first, although crucial, step towards removing systematic errors in atomic input data required for quantitative stellar spectroscopy. We thoroughly assess the quality of fundamental atomic data available in the largest repositories by comparing very high-quality observed stellar spectra with state-of-the-art theoretical spectra. Whereas this type of study has currently been carried out for very few stars at the time, and mostly limited to comparable spectral types assembled from various sources, we will combine, analyze, and offer the community the first uniform large collection of benchmark and reference stars. The results will be more complete than any other to date in terms of coverage of the stellar parameter space, as well as the spectral wavelength coverage. The impact of BRASS will be multiple. The high-quality input spectra and output datasets (e.g. fully quality-assessed atomic data) will be offered in the public domain. They will be unique by size, quality, and coverage of the stellar parameters space, and consequently benefit a large array of astrophysical research topics, including important feed-back for atomic physics.

In this paper we provide an overview of our research activi- 
ties between Dec. 2014 and mid 2016 for BRASS. We present new results of a pipeline we implemented for accurate astrophysical parameter (APs) determinations using detailed synthetic spectrum calculations. We determine the APs of 5 BRASS benchmark stars of F, G, K, A, and B spectral types observed with signal-to-noise ratios of $\sim 1000$. In Sect. 2 we summarize the observation and calibration of the BRASS spectra. Section 3 provides an overview of new software developments for the determination of atmospheric conditions and radial velocities with BRASS spectra. Section 4 presents a brief overview of recent BRASS Data Interface (BDI) developments currently offering online the observed and theoretical BRASS spectra of the Sun and $51 \mathrm{Peg}$, combined with confirmed line identifications and the retrieved atomic input data. In Sect. 5 we discuss the selection of a subset of atomic lines between $4000 \AA$ and $6800 \AA$, also marked in the BDI, with minimal blending in the synthetic spectra. We summarize the pipeline preparations for the planned atomic data quality testing of selected unblended lines and give an example of the Fe II $\lambda 5264$ line in the solarlike benchmark star 51 Peg (G2.5).

\section{Observations}

\subsection{BRASS benchmark stars}

We observed benchmark spectra of $~ 20$ BAFGK-type stars with the HERMES instrument of the Belgian $1.2 \mathrm{~m}$ Mercator telescope at the Roque de los Muchachos Observatory on La Palma Island (Canary Islands, Spain). HERMES (High Efficiency and Resolution Mercator Echelle Spectrograph) is a high-efficiency prism-cross-dispersed fibre-fed bench-mounted échelle spectrograph that observes the complete wavelength range from $4200 \AA$ to $9000 \AA$ in a single exposure [9]. We used the High Resolution Fiber mode $(R=85,000)$ for 10 to 50 concatenated observations of at most 1800 seconds, that are co-added for increasing the $\mathrm{S} / \mathrm{N}$ ratio of the final spectrum.

The benchmark star observations for BRASS yielded very high $\mathrm{S} / \mathrm{N}$ spectra ( 800-1200) in dedicated HERMES programs since 2009. The benchmark stars are brighter than $7^{\mathrm{m}} .0$ in $V$ in order to achieve the very high $\mathrm{S} / \mathrm{N}$ ratios. The stars have been selected because the spectral lines are narrow. The line rotational broadening or $v \sin i$-values are typically below $25 \mathrm{~km} \mathrm{~s}^{-1}$ for B-stars, and $<13 \mathrm{~km} \mathrm{~s}^{-1}$ for the AFGK-type stars. These benchmark stars have been carefully selected avoiding possible binaries. They do not belong to known stellar variability classes and are not chemically peculiar stars or metal-poor stars.

The high-resolution spectrum of the Sun was observed in 1981 with the NSO/KPNO Fourier Transform Spectrograph (FTS) at the McMath-Pierce Solar Facility. The spectral resolution of the FTS flux spectrum is $R \sim 350,000$, with nominal $\mathrm{S} / \mathrm{N}$ ratios of $\sim 2500$. More information about the calibration of the solar intensity (averaged over the solar disk) atlas is provided in [10].

The spectra were calibrated using the latest version of the HERMES pipeline (release V6.0) developed at the Royal Observatory of Belgium and the University of Leuven, in collaboration with the HERMES Consortium. The typical calibration steps are performed, including spectral order tracing and extraction, average flat-fielding, Th-Ar lamp wavelength calibration, and cosmic ray hit removal using cross-order profiling.
BRASS includes $\sim 10$ stars observed with the Very Large Telescopes Ultraviolet and Visual Echelle Spectrograph (VLTUVES) in the Southern Hemisphere (SH). The calibrated UVES spectra are offered in the ESO Data Archive. The Paranal Observatory Project (ESO-POP) catalogue offers high-resolution and high S/N ratio UVES spectra $(\sim 300-500)$ of SH objects observed in the wavelength range 3040 - 10,400 $\AA$ [11]. However, many POP archive products were processed using nonoptimal calibration images of a database of calibration frames that is currently out of date. In the best cases it offers suboptimal quality spectra, and for worse cases, the frames have introduced spurious artefacts and incorrect wavelength shifts in the final spectra.

We have re-processed and re-calibrated the UVES-POP spectra for the goals of the BRASS project. We retrieved the uncalibrated POP observations from the ESO Archive and collected all calibration frames observed closest in time to the selected POP observations. Next, we re-calibrated the raw POP science data using the latest UVES pipeline version (v5.5.7) and compared the results with the spectra of the POP archive which used UVES pipeline v1.2.0. Our detailed comparison considered the S/N ratios of the final UVES spectra, the wavelength scale, the amplitude of spurious flux ripples, and the quality of merging the échelle orders. The results were also compared to spectra of the ESO External Data Product (EDP) Archive recently calibrated by ESOs QC/Archive Group using the Basic Master Database of basic parameters. The EDP spectra have been provided by ESO as 'science-ready' spectra to the scientific community.

The UVES-POP wavelength range of 3000-10,000 $\AA$ is divided by 4 instrumental setups with 1 to 3 exposures per setup, observed with a resolving power of $R=50,000-70,000$. It is important to point out that the original POP calibrations combined all exposures. For the BRASS re-calibrations we however did not combine the exposures and re-calibrated all spectra individually. It allows us, for example, to check for potential intrinsic stellar variability. We find that using the same calibration algorithms as POP the BRASS re-calibrated spectra show major improvements in the UVES flux ripples and échelle order merging. The flux ripples are $\sim 5$ times weaker in the recalibrated spectra. This allows us to calculate order-merged spectra, while the POP spectra can only be used order by order. The comparison of radial velocities reveals a difference of $13-15 \mathrm{~km} \mathrm{~s}^{-1}$ between the BRASS re-calibrated spectra and the POP spectra because the barycentric velocity was not applied to our data. After barycentric correction the radial velocities are identical. The RMS error of our UVES wavelength solution is $106 \mathrm{~m} \mathrm{~s}^{-1}$. The comparison of the 'manually' recalibrated UVES-POP spectra with those provided in the ESO EDP Archive shows that the spurious flux ripples are properly corrected in both cases, while the $\mathrm{S} / \mathrm{N}$ ratios are $15 \%-50 \%$ smaller compared to our results.

\subsection{BRASS reference stars}

The quality assessments of the fundamental atomic data for BRASS will be primarily performed with benchmark stars of $\mathrm{S} / \mathrm{N} \sim 800-1200$. For observational and practical reasons, this set of benchmarks must however be limited to about 30 stars that uniformly, although rather sparsely, samples the BAFGKtypes. 
For the goals of BRASS we substantially increased the benchmark dataset in order to fully explore the diversity of spectral properties in the 5 spectral types. The science goals place stringent requirements on the observed datasets we call the reference spectra of BRASS. It requires more than one hundred spectra combining wide wavelength coverage (4000 $\AA$ $6800 \AA)$, high spectral resolution $(R>80,000)$ and large to very high $\mathrm{S} / \mathrm{N}$ ratios $(>200-1000)$ over all spectral types between $\mathrm{B}$ and $\mathrm{K}$, e.g., as uniformly as possible covering the $\mathrm{T}_{\text {eff }}{ }^{-}$ range from $\sim 4000 \mathrm{~K}$ to $15-20 \mathrm{kK}$. Such an extensive spectral dataset is currently not available in the public domain.

As part of HERMES Program 28 (P28), we have observed since 2010 the spectra of the brightest ten stars of every spectral type, each subtype, and luminosity class in the Northern Hemisphere (NH), with S/N ratios exceeding 200 in all cases. The program currently contains 3416 spectra of 1961 unique objects. The resulting data archive contains a very extensive collection of high-quality spectra, covering the entire range of stellar parameters (including in metallicity $[\mathrm{M} / \mathrm{H}], v \sin i$, etc.) All targets have $V<8^{\mathrm{m}} .5$, while the catalogue is complete below $V=4^{\mathrm{m}}$, for declinations above $-30^{\circ}$.

To ensure that BRASS uses the highest quality spectra available we filtered the $\mathrm{P} 28$ database according to $\mathrm{S} / \mathrm{N}$ ratio. $\mathrm{S} / \mathrm{N}$ was estimated at 5 effective wavelengths ( $U, B, V, R, I$-bands) and good quality spectra with $\mathrm{S} / \mathrm{N}>100$ in 3 of the 5 wavelength bands were selected. The resultant P28 database contains 2092 good quality spectra of 1796 unique objects. Around $20 \%$ of the database is comprised of objects with more than two observations.

\section{Astrophysical Parameter determinations}

\subsection{Atmospheric conditions}

In 2015 we developed a suite of computer codes (BRASSAPS) for the semi-automatic determination of APs and chemical composition of the BRASS benchmark and reference spectra. It requires three subsequent major computational steps. First the pre-processor estimates the APs using a limited number of diagnostic $\mathrm{H}$ Balmer, $\mathrm{Fe}$, and $\mathrm{Mg}$ absorption lines. The second pipeline step iterates over $\mathrm{T}_{\text {eff }}$, surface gravity $(\log g)$, microturbulence velocity $\left(\zeta_{\mu}\right)$, and $[\mathrm{M} / \mathrm{H}]$ until the best fit is found to the detailed shapes of a more extensive set of diagnostic photospheric lines (e.g., $\sim 40$ selected Fe I and Fe II lines) having reliable atomic data values of line $\log (g f)$, energy levels $(\chi)$, rest wavelength $\left(\lambda_{0}\right)$, and Einstein coefficient $\left(A_{k i}\right)$. The final step uses the iterated APs as input for measuring individual element abundances $([\mathrm{X} / \mathrm{H}])$ from selected sets of sufficiently strong (medium-strong to strong) lines on the curve of growth (COG).

The synthetic spectra are computed in LTE using hydrostatic, plane-parallel atmosphere model grids we iterate using ATLAS9 [12]. The models adopt the new Opacity Distribution functions of [13]. We use a constant mixing-length parameter of 1.25 for convection and omit overshoot or turbulent pressure contributions. We calculated a large homogeneous grid of synthetic spectra between $3200 \AA$ and $6800 \AA$ using the SCANSPEC code ([14]). The parameter space of applicability of the BRASS APs pipeline currently is: $4 \mathrm{kK}<\mathrm{T}_{\text {eff }}<15$ $\mathrm{kK}$ in steps of $50 \mathrm{~K} ; 0.0<\log g<5.0 ; 0 \mathrm{~km} \mathrm{~s}^{-1}<\zeta_{\mu}<$ $20 \mathrm{~km} \mathrm{~s}^{-1} ;-5.0<[\mathrm{M} / \mathrm{H}]<1.0$, and $0 \mathrm{~km} \mathrm{~s}^{-1}<v \sin i<$
$300 \mathrm{~km} \mathrm{~s}^{-1}$. Important line broadening effects for strong resonance lines and hyperfine splitting of odd atomic numbers are included in the synthetic spectrum calculations. For the Sun we adopt the chemical composition of [15]. The equation of state also incorporates many di-atomic molecules with updated partition functions for important hydrides such as $\mathrm{CH}, \mathrm{AlH}, \mathrm{CaH}$, $\mathrm{SiH}, \mathrm{FeH}, \mathrm{CrH}, \mathrm{NH}, \mathrm{OH}, \mathrm{MgH}$, carbon bearing molecules $\mathrm{C}_{2}$, $\mathrm{CN}, \mathrm{CO}$, and the oxides $\mathrm{TiO}, \mathrm{ZrO}$, and $\mathrm{VO}$. We retrieved extensive molecular line data values in more recent literature and in the online ExoMol database providing several millions of extra lines. These data have been organized in the BRASS database and incorporated in the atomic input line lists for our detailed synthetic spectrum calculations.

The line-of-sight microturbulence velocity $\zeta_{\mu}$ is assumed to be independent of depth in the BRASS atmosphere models of the dwarf and subgiant benchmark stars. Note that the five APs are methodically varied and iterated. For example $\zeta_{\mu}$ is measured from the BRASS spectra and not simply parameterized through $\mathrm{T}_{\text {eff }}$ and $\log g$ because this is not possible for A- and B-type stars $\left(\mathrm{T}_{\text {eff }}>7000 \mathrm{~K}\right)$. There is a maximum in the projected microturbulence velocity around the mid A-type stars [16]. The $v \sin i$-value is accurately iterated in steps of $1 \mathrm{~km} \mathrm{~s}^{-1}$ for obtaining the best fit to the detailed (broadened) shapes of the diagnostic metal lines.

The observed BRASS spectra are automatically continuum flux normalized using a special 'template normalization' procedure. It searches for variable wavelength points over sufficiently continuous flux regions close to the stellar continuum level in the theoretical spectra for folding the observed spectra to these (continuum anchor) points. The re-normalization step repeats every time the APs are updated during the best fit iterations procedure. It ensures that local flux normalization effects become minimized and the continuum normalization around the lines we select for evaluating the quality of atomic line data is consistent with the APs we determine from the diagnostic lines set.

Figure 1 shows a plot of $\sim 30$ benchmark stars with BRASS HERMES and UVES spectra. The Sun and names of 5 stars of BAFGK-types are marked. The APs we determine from the HERMES spectra of these stars are listed in Table 1. We also compared them to the APs of previous high-resolution observations in the literature. Figure 2 shows the best fit to the spectrum of the Sun and Procyon observed between $5569 \AA$ and $5583 \AA$ (solid black drawn lines). The marked medium-strong neutral metal lines are best fit (red lines) with the atomic data values listed below the lines in the bottom panel. The atomic data values are selected from the literature and/or retrieved in atomic databases for calculating the best fit to the line depths and shapes. The best fits with these values are good to excellent (e.g., $\leq 0.01$ in normalized line depths). The green lines mark the spectra we calculate without the neutral lines. The lines are blended with weak atomic or molecular background lines below $5 \%$ of the line equivalent widths.

Figure 3 shows a similar plot for the wavelength region between $4514 \AA$ and $4525 \AA$ in A-type benchmark star 68 Tau, and in late B-type benchmark star HR 7512. The $\log (g f)$-values of three Fe II lines observed in both hot stars are marked with different colors. We retrieve $\log (g f)$-values for the Fe II $\lambda 4515$ line in NIST (N), SpectroWeb (SpW), VALD-2 (V-2), and VALD $-3(\mathrm{~V}-3)$. The values between -2.450 and -2.540 correctly fit 
the observed line profile. For the Fe II $\lambda 4521$ line we find the best fit for $\log (g f)=-2.600(\mathrm{~N})$ and $-2.617(\mathrm{~V}-2)$, while the value of $\log (g f)=-2.983(\mathrm{SpW})$ is significantly too small and the theoretical line depth underestimates the observed depth of the central core. For the Fe II $\lambda 4522$ line, on the other hand, we retrieve four $\log (g f)$-values that significantly overestimate the central core depth. We find the same relative flux differences for HR 7512 in the bottom panel, however for $\mathrm{T}_{\text {eff }}$ of over 2000 $\mathrm{K}$ warmer than $68 \mathrm{Tau}$.

\subsection{Radial velocity}

We developed a new computer code (BRASSVRAD) in 2015 for measuring accurate radial velocities of BRASS benchmark and reference stars. It employs a $\chi^{2}$-minimization method of the detailed shapes and positions of observed spectral lines using parameterized template fit functions. The sample of observed lines is carefully selected over a broad range of wavelengths between $4000 \AA$ and $6800 \AA$.

We have carried out a variety of tests for assessing the accuracy of our radial velocity results. The measurement method does not require a 'mask' for cross-correlation with the observed spectra. We also collected complementary spectra from various online public databases (OHP-SOPHIE, OHP-ELODIE, PdM-NARVAL) for combination with the BRASS reference spectra. Based on measurements with BRASSVRAD we compiled a list of $\sim 40$ A- \& B-type reference stars having radial velocity values that stay constant within $\sim 0.5$ to $1.0 \mathrm{~km} \mathrm{~s}^{-1}$. For a good number of stars the constant radial velocity values have also been tested using the complementary spectra observed before 2010. The HERMES spectra of the Vrad-constant stars will be incorporated in the BDI and will be very useful in the scientific community as warm radial velocity standard stars observed with high signal-to-noise ratios and high spectral resolution. They will benefit the astrophysics community at large, in particular for analysis of spectra from the ESA-Gaia mission because they include the Radial Velocity Spectrometer wavelength region as well.

We find good agreement between published Vrad-values and the values we compute with BRASS spectra. The Vrad differences typically increase towards larger $v \sin i$-values. A comparison of Vrad-values we calculate for 5 A-stars with BRASSVRAD and with a classical CCF method using a cross-correlation mask reveals small differences below $2 \mathrm{~km} \mathrm{~s}^{-1}$ in case $v \sin i \simeq$ $70 \mathrm{~km} \mathrm{~s}^{-1}$. Further testing demonstrates that our radial velocity measurement method using selected spectral lines is very precise. We find that the code can detect small (Doppler) effects of changes in Earth's atmospheric pressure that typically stay below $\sim 500 \mathrm{~m} \mathrm{~s}^{-1}$. A more detailed description of these results will be given elsewhere.

We investigated HERMES spectra of BRASS benchmark star $o$ Peg (A1 IV). We find the white subgiant reveals a steady decrease of Vrad of $\simeq 1.7 \pm 0.3 \mathrm{~km} \mathrm{~s}^{-1}$ in 14 HERMES observations between 2009 and 2014. In the literature $o$ Peg is currently not known to be a binary or variable star. We propose $o$ Peg is a long-term binary with $P_{\text {orb }} \sim 10 \mathrm{yr}$.

The white giant $\alpha$ Sextantis (A0 III) reveals small Vrad variability of $\sim 0.5 \mathrm{~km} \mathrm{~s}^{-1}$ over a period of $\sim 200 \mathrm{~d}$. We observe an overall decreasing trend in Vrad of $\sim 1 \mathrm{~km} \mathrm{~s}^{-1}$ from 206 HERMES observations between 2010 and 2015. HERMES observations of BRASS reference star 13 Mon (A0 I) also show small changes (1-2 $\mathrm{km} \mathrm{s}^{-1}$ ) of Vrad between 2010 and 2014. We observe short-term variability of $\sim 1.5 \mathrm{~km} \mathrm{~s}^{-1}$ over about one month, possibly due to stellar pulsations of this white supergiant.

\section{BRASS Data Interface}

In 2016 we developed a new user interface (BDI) for online querying and visualization of BRASS spectra and atomic line data. The first version of the BDI at brass.sdf .org, shown in Fig. 4, loads in every modern internet browser. User interactions combine fast spectrum display and atomic data listing using integrated Hypertext (HTML), JavaScript, JQuery (CSS), and Dygraphs software technology. The BDI development is targeted at user-friendliness, and ensures easy visualization, selection and retrieval of any desired subset of the BRASS database. The BDI functionality is expanded from SpectroWeb [17] (spectra.freeshell.org) for example also allowing synchronized wavelength zooming on 4 spectral segments of $30 \AA$ for various selected stars.

The BDI currently offers the BRASS benchmark spectra of the Sun and 51 Peg (fall 2016). In Fig. 4 the lines marked with blue labels can be mouse-clicked for listing atomic line data in the right-hand panels. The atomic data values are collected from the literature and various online atomic data repositories (VAMDC, NIST, SpectroWeb, VALD-3, VALD-Moscow [18], CHIANTI [19], Spectr-W3 [20], TOPbase [21]). The theoretical unbroadened spectra are shown in blue color. The atomic data list also provides the calculated (unbroadened) depth of the line in case it exceeds $2 \%$ of the continuum normalized flux level shown at unity.

In Fig. 4 the red labels mark lines we tested for blending with background lines in the synthetic spectrum calculations. The lines in the Sun (top panel) are selected (i.e., labelled and numbered in red) in case the equivalent width of the line overlaps $<5 \%$ with weaker atomic and/or molecular background lines. It yields 902 'unblended' lines between $4200 \AA$ and $6800 \AA$. The atomic data and percentages of line blending are listed in red with a single mouse-click on the line label. For example, three Fe I and Fe II lines with label numbers 360, 361 , and 362 are shown in the Sun. The normalized central line depths we compute in the broadened synthetic spectrum are also listed. Note that the BDI graphics panels are mousesensitive and display the line identifications and rest wavelengths by simply hovering over the dynamic line labels. The labels can be shifted Up and Down the graphs with the 'Shift labels' buttons to avoid dense label overlay when zooming out.

The bottom panel of Fig. 4 shows the spectral region around the Fe II $\lambda 5264$ line in 51 Peg. The Fe II line blends by $1.72 \%$ with a very weak Co I $\lambda 5264$ line having a normalized central depth of 0.02 . The Fe II line has maximum calculated depth of 0.419 and is a medium-strong line on the COG. A doubleclick on the red line label marks it in yellow and lists the atomic line data and properties in green in the right-hand panels. The green list can be supplemented with data of more selected lines of interest. Users can save the list to a local hypertext file with a simple button click on top. The BDI will link the repository pages with atomic line data quality test results currently under construction. 
Table 1. Five BAFGK-type benchmark stars observed with Mercator-HERMES with S/N-ratios of 800-1200 between $4000 \AA$ and $9000 \AA$ for which we determine atmospheric parameters for BRASS.

\begin{tabular}{rrrrlrrrrr}
\hline Name & HD & $\begin{array}{r}V \\
(\mathrm{mag} .)\end{array}$ & SpT & Lum. class & $\begin{array}{r}\mathrm{T}_{\text {eff }} \\
(\mathrm{K})\end{array}$ & $\begin{array}{r}\log (g) \\
(\mathrm{dex})\end{array}$ & $\begin{array}{r}{[\mathrm{M} / \mathrm{H}]} \\
(\mathrm{dex})\end{array}$ & $\begin{array}{r}\zeta_{\mu} \\
\left(\mathrm{km} \mathrm{s}^{-1}\right)\end{array}$ & $\begin{array}{r}v \sin i \\
\left(\mathrm{~km} \mathrm{~s}^{-1}\right)\end{array}$ \\
\hline HR 7512 & 186568 & 6.057 & B8 & III & 11070 & 3.44 & -0.50 & 2.00 & 18.0 \\
68 Tau & 27962 & 4.31 & A2 & IV-V & 8810 & 3.80 & 0.13 & 2.40 & 11.8 \\
Procyon & 61421 & 0.34 & F5 & IV-V & 6550 & 4.00 & 0.00 & 1.80 & 3.9 \\
51 Peg & 217014 & 5.46 & G2.5 & IVa & 5804 & 4.42 & 0.20 & 1.10 & 4.0 \\
Sun & - & -26.74 & G2 & V & 5777 & 4.44 & 0.00 & 1.10 & 2.5 \\
Arcturus & 124897 & -0.04 & K1.5 & III & 4100 & 2.00 & -0.50 & 1.70 & 5.5 \\
\hline
\end{tabular}

\section{Discussion}

We retrieved the atomic line data values from the literature and online atomic databases such as NIST and various databases offered in VAMDC. The VAMDC portal offers important research infrastructure for querying and retrieving atomic line data according user-defined selection criteria from a large variety of repositories of astrophysical interest. The homogenization of these line data to make them more easily comparable is in progress. We developed a novel method for crossmatching the databases searching for identical atomic line transitions with a sophisticated method that compares the upper and lower electronic state configurations and angular momenta $J$. The new method yields multiple occurrences of atomic data values of identical lines offered by the different data providers. We developed the method for BRASS to ensure the cross- matching of atomic line transitions is 'exact' and based on the precise electronic state configurations. It eliminates possible errors of 'inexact' cross-matching methods limited to the line rest- wavelengths and energy levels (hence ignoring the detailed electronic configuration and term information when available). The new cross-matching method is very useful for removing duplicate occurrences of the same lines from hetrogeneous linelist compilations required for the advanced synthetic spectrum calculations. We have retrieved the atomic data values of $\sim 184,000$ line transitions from 6 online repositories. We systematically queried, cross-matched and organized these atomic data per spectral line in BRASS (further detailed in [22]) for state-of-the-art synthetic spectrum calculations.

We investigated the amount of line blending in the 6 BRASS benchmark stars of Table 1 . The lines are pre-selected before the quality of fit assessments against the observed benchmark spectra of BAGFK-types. Over 1200 'sufficiently clean' lines have so far been identified across the 5 spectral types. Towards the cooler G- and K-type stars the molecular lines dominate the amount of line blending. Towards the hotter B- and A-type stars the depths of the neutral lines rapidly decrease and a relatively smaller number of 'unblended' ionic lines is selected. We find the maximum number of clean lines in Procyon (F5) with about 3-4 times more selected lines compared to Arcturus (K1.5) and HR 7512 (B8).

The Fe II line at $5264.802 \AA\left(\chi_{\text {low }}=3.23 \mathrm{eV}\right)$ in the middle panel of Fig. 4 (bottom graph) provides an excellent example of the atomic data quality assessments we plan for BRASS using selected sets of 'clean' spectral lines in the BAGFK benchmark stars. 51 Peg is the first main-sequence star with a Jupitersize exoplanet discovered in 1995 [23]. Since the discovery the star has been relatively well-studied with over 20 publications of APs determined for the host star using high-resolution spectroscopic observations. $\mathrm{T}_{\text {eff }}$-values ranging from $5674 \mathrm{~K}$ to $5804 \mathrm{~K}$ have been published since 2005 . The largest $\mathrm{T}_{\text {eff }^{-}}$ value corresponds to $[\mathrm{M} / \mathrm{H}]=0.24 \mathrm{dex}$ and the smallest to 0.18 dex. We compute the broadened theoretical line profile of the Fe II $\lambda 5264$ line adopting these APs.

In Fig. 5 we find the best fit (red solid line) to the Fe II line profile observed in $51 \mathrm{Peg}$ using $\log (g f)=-3.130$ of [24] for $\mathrm{T}_{\mathrm{eff}}=5804 \mathrm{~K}$ and $[\mathrm{M} / \mathrm{H}]=0.20$ (see Table 1 ). In case we use $\mathrm{T}_{\text {eff }}=5674 \mathrm{~K}$ and $[\mathrm{M} / \mathrm{H}]=0.18$ the calculated line depth decreases by $\sim 0.02$ (blue line). The decrease of $130 \mathrm{~K}$ in $\mathrm{T}_{\text {eff }}$ has a limited effect on the line depth. However, if we use $\mathrm{T}_{\text {eff }}=5804$ $\mathrm{K}$ and the $\log (g f)$-value of -3.23 provided in NIST ([25]) the calculated line depth decreases more significantly by $\simeq 0.05$ (short-dashed drawn line). The theoretical line depth decreases further by $\sim 0.09$ compared to the observed line depth in case we use $\log (g f)=-3.303$ (VALD-2 [26]; theoretical value retrieved in 2005 for SpectroWeb). These differences of published $\log (g f)$-values are appreciable (i.e., $\simeq 0.17$ dex) and yield considerably different calculated line depths and equivalent line widths.

The S/N ratios of the BRASS benchmark spectra are sufficiently high $(\sim 1000)$ for determining the fit quality of line profiles we calculate for various atomic input values from different data providers. The BRASS project will assess the accuracy of the atomic data values (primarily $\log (g f), \lambda_{0}$, and $\mathrm{A}_{\mathrm{ki}}$ ) in the synthetic spectrum calculations by comparing the line fit quality in a number of carefully selected benchmark stars belonging to the same spectral types in Fig. 1.

\section{Summary and outlook}

The BRASS benchmark and reference spectra will be published and provided in the BRASS website by the end of 2018. The BDI will offer the complete HERMES and UVES spectra for downloading. For the benchmark stars we will also include the theoretical (un-broadened and broadened) spectra. The lists of unblended atomic lines and all the retrieved atomic data per spectral type, together with our line data accuracy assessments, will be published and incorporated with supporting tables and graphs in the BDI.

\section{Acknowledgements}

The research for the present results has been subsidized by the Belgian Federal Science Policy Office under contract No. BR/143/A2/BRASS. The HERMES spectrograph is installed at the Mercator Telescope, operated on the island of La Palma by the Flemish Community, at the Spanish Observatorio del 
Roque de los Muchachos of the Instituto de Astrofsica de Canarias and supported by the Fund for Scientific Research of Flanders (FWO), Belgium, the Research Council of KU Leuven, Belgium, the Fonds National de la Recherche Scientifique (F.R.S.- FNRS), Belgium, the Royal Observatory of Belgium, the Observatoire de Genève, Switzerland and the Thüringer Landessternwarte Tautenburg, Germany.

\section{References}

1. M L Dubernet, B K Antony, Y A Ba, et al., J. Phys. B: At. Mol. Opt. Phys. 49074003 (2016)

2. A Kramida, Y Ralchenko, J Reade, and the NIST ASD Team, NIST atomic spectra database (version 5) (2016) www.nist.gov/pml/atomic-spectra-database

3. J Tennyson, and S N Yurchenko, Mon. Not. R. Astron. Soc. 425 21 (2012) www . exomol. com

4. European Space Agency, Gaia Science Performance, 2017 www. cosmos.esa.int/web/gaia/science-performance

5. A Lobel, J. Phys. Conf. Ser. 328012027 (2011)

6. Y S Lee, T C Beers, T Sivarani, et al., AJ 1362050 (2008)

7. A Siebert, M E K Williams, A Siviero, et al., AJ 141187 (2011)

8. J B Sousa, N C Santos, V Adibekyan, et al., A\&A 56121 (2014)

9. Raskin, H van Winckel, H Hensberge, et al., 2011, A\&A 526 A69 (2011)

10. H Neckel, and D Labs, Solar Phys. 90205 (1984)

11. S Bagnulo, E Jehin, C Ledoux, et al., Messenger 11410 (2003)

12. R L Kurucz, Rev. Mexicana AyA 2345 (1992)

13. F Castelli, and R Kurucz R, IAU Symp. 210, Modelling of Stellar Atmospheres, Uppsala, Sweden, eds. N E Piskunov, W W Weiss and D F Gray, ASP-S210 (2003)

14. A Lobel, Can. J. Phys. 89395 (2011)

15. N Grevesse, M Asplund, and A J Sauval, Space Science Reviews 130105 (2007)

16. H Bruntt, S Basu, B Smalley, et al., MNRAS 423122 (2012)

17. A Lobel, J. Phys. Conf. Ser. 1300120215 (2008)

18. T Ryabchikova, N Piskunov, R L Kurucz, et al., Phys. Scr. 90 054005 (2015)

19. P Young, K Dere, E Landi, G Del Zanna, and H Mason, Journal of Physics B: Atomic, Molecular and Optical Physics 497 (2016) www . chiantidatabase .org

20. A Y Faenov, et al., Proc. 3rd Int. Conf. on Atomic and Molecular Data and Their Applications 253 (2002) spectr-w3.snz.ru

21. W Cunto W, C Mendoza, F Ochsenbein, C J Zeippen, A\&A 275 L5 (1993) cdsweb.u-strasbg.fr/OP.htx

22. M Laverick, A Lobel, P Royer, C Martayan, and T Merle, Can. J. Phys. (2017), submitted for this volume

23. M Mayor, and D Queloz, Nature 378355 (1995)

24. J Meléndez, and B Barbuy, A\&A 497611 (2009)

25. R Schnabel, M Schultz-Johanning, and M Kock, A\&A 4141169 (2004)

26. F Kupka, N E Piskunov, T A Ryabchikova, H C Stemples, and W W Weiss, A\&ASS 139119 (1999) 


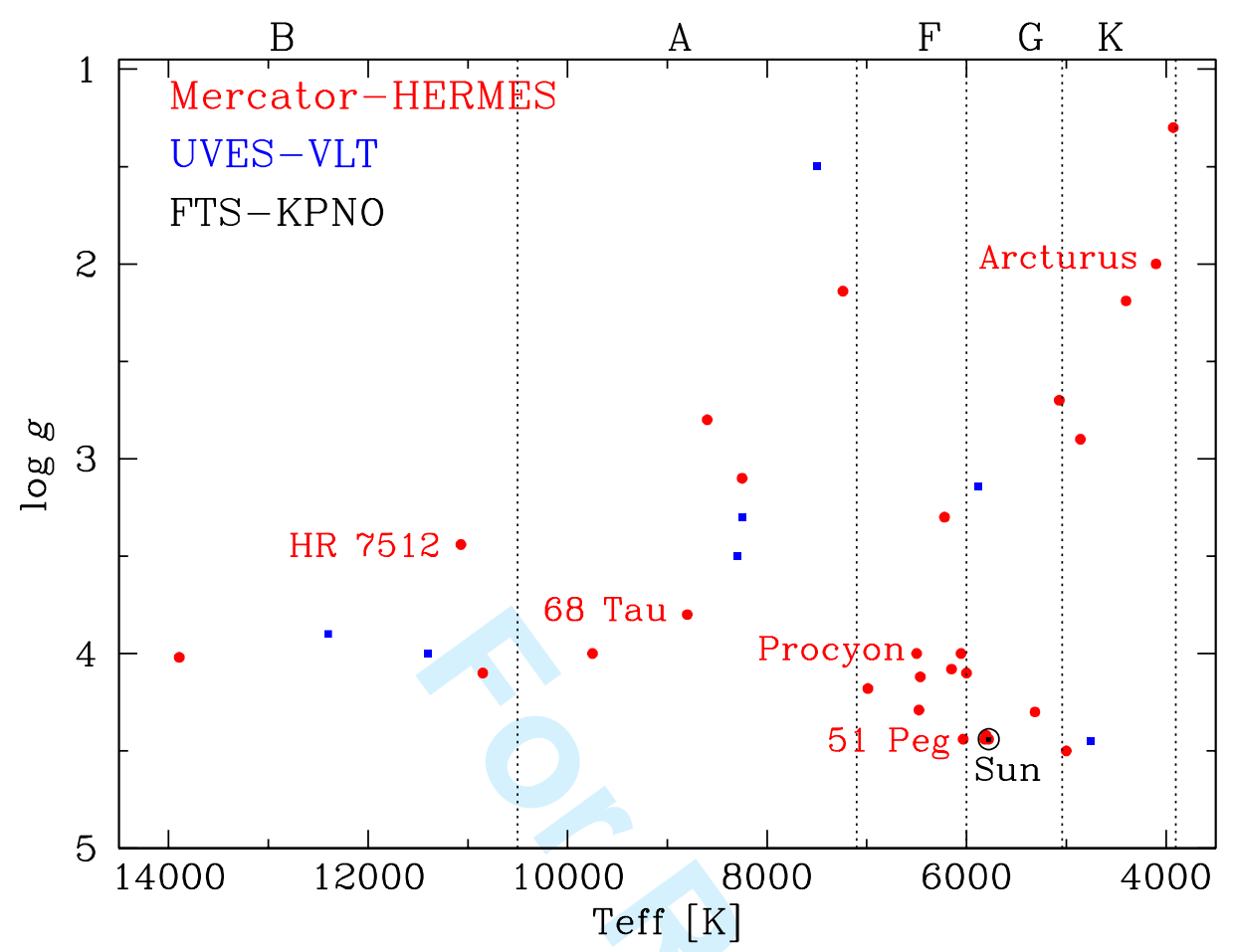

Fig. 1. Effective temperature $\left(\mathrm{T}_{\text {eff }}\right)$ vs. surface gravity $(\log g$ )-diagram of $\sim 30$ BAFGK benchmark stars observed for BRASS with Mercator-HERMES (red dots) and VLT-UVES (blue squared symbols). The HERMES spectra have S/N ratios of $\sim 800-1200$ and have been observed since 2009. The UVES spectra have S/N 300-500 and have been observed in the SH for the ESO-POP Archive before 2001. The marked stars have accurate astrophysical parameters in Table 1 we determine with the BRASS benchmark spectra. The vertical dotted lines mark the division between the 5 spectral types (see text). 


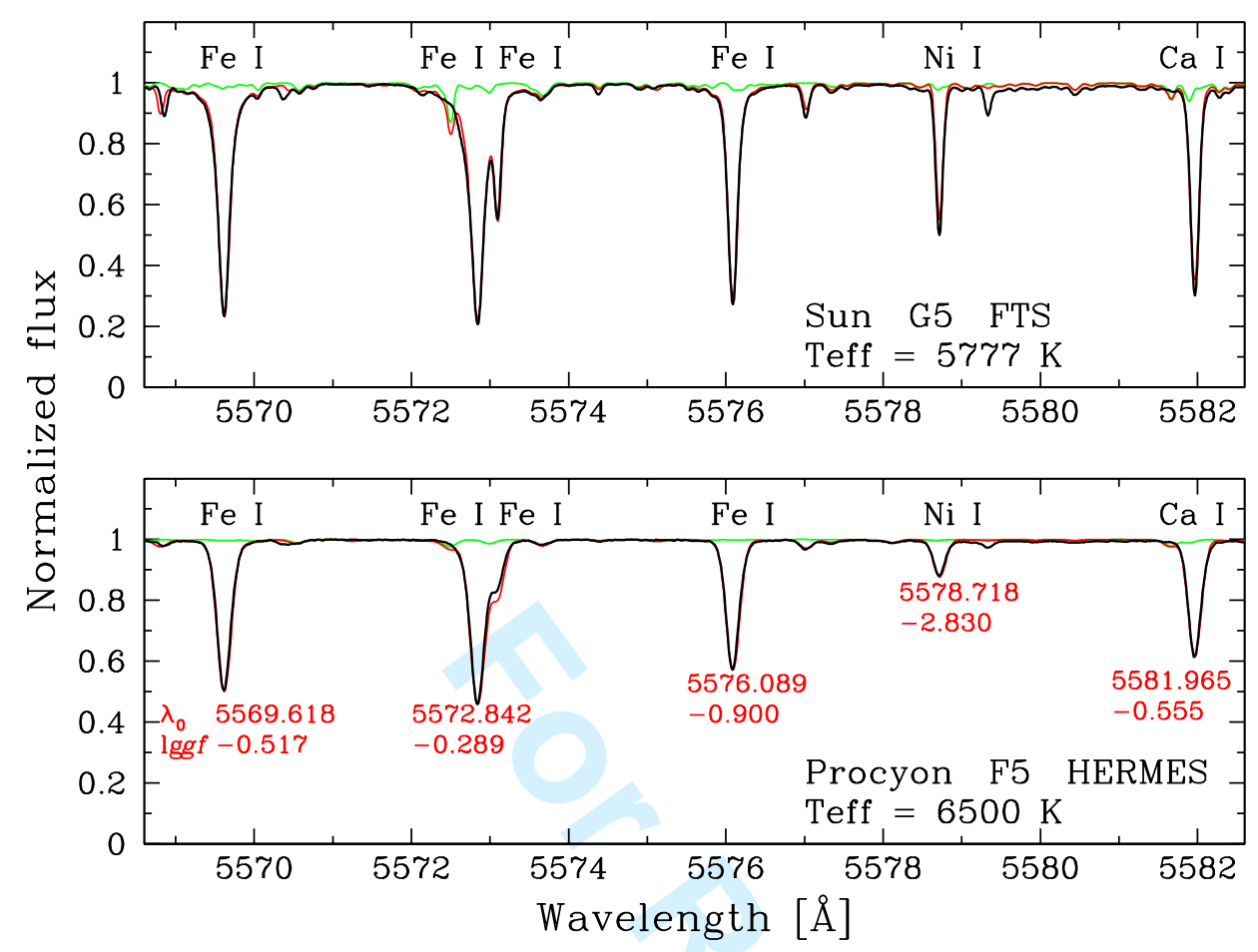

Fig. 2. The BRASS observed benchmark spectra (black lines) of the Sun (top panel) and Procyon (bottom panel) are shown over $\sim 15 \AA$ with marked medium-strong neutral metal absorption lines. The theoretical spectra we calculate with published atomic line data values of $\lambda_{0}$ and $\log (g f)$ are listed below the lines in the bottom panel and provide the best fit (red lines) to the observed spectra. The green drawn lines show the synthetic spectra we calculate without these neutral lines. They reveal the lines are not significantly blending together with strong background lines that can complicate the best line fit procedure and the proper selection of atomic input data. 


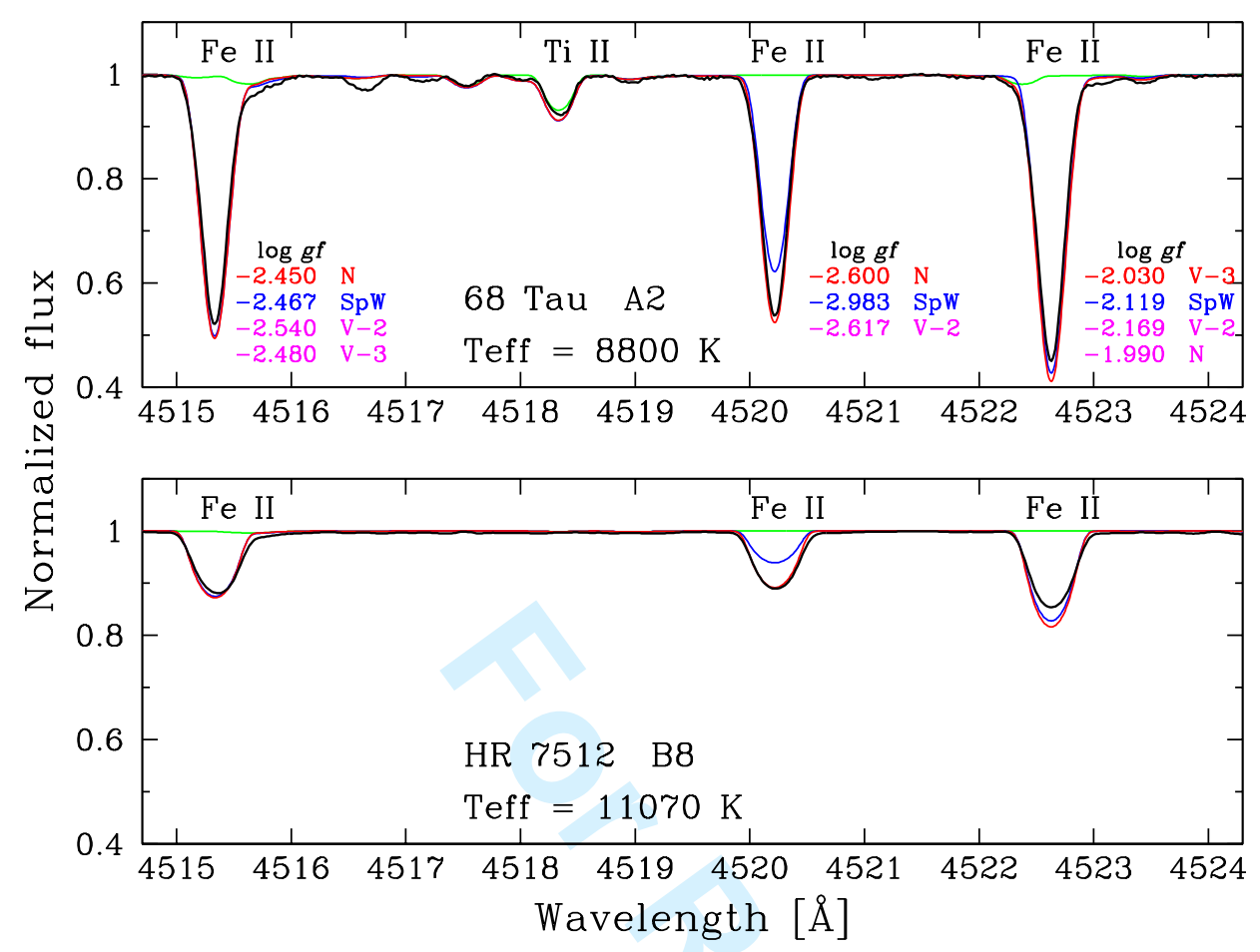

Fig. 3. The BRASS observed benchmark spectra (black lines) of 68 Tau (top panel) and HR 7512 (bottom panel) are shown over $\sim 10 \AA$ with three Fe II absorption lines. The theoretical spectra we calculate for various published $\log (g f)$-values are marked with colors below the lines. The values of the Fe II $\lambda 4515$ line are similar and fit the observed line depths in both stars although HR 7512 is over $2000 \mathrm{~K}$ hotter than 68 Tau (see text). 


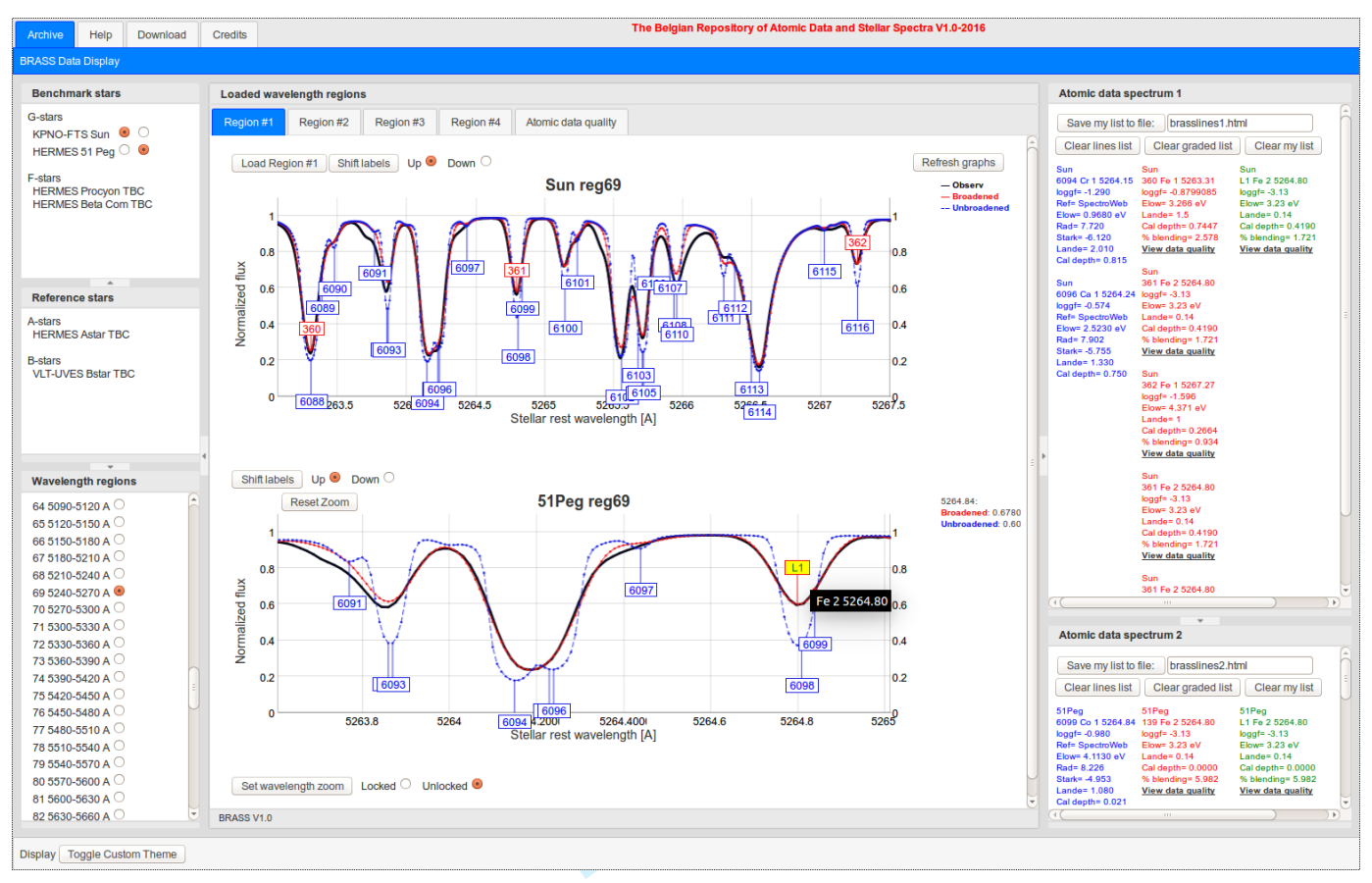

Fig. 4. The BRASS Data Interface (BDI development version 1.0 of fall 2016) showing the BRASS benchmark spectrum of the Sun (top panel) and the solar-like dwarf 51 Peg (bottom panel). The observed (black dotted lines) and theoretical spectra (blue and red dotted lines) can be simultaneously loaded into 4 Region tab pages over $30 \AA$ wavelength intervals selected in the left-hand bottom panel. The dynamical graphs can be synchronously wavelength zoomed. Blue labels mark identified spectral lines, while red labels are selected lines that blend by $<5 \%$ with background lines. The line labels are mouse-sensitive and can be single- and/or double-clicked. Mouse interaction with the labels displays the atomic line data in the right-hand panels for local storage by the user. Planned updates of the BDI will incorporate all BRASS benchmark and reference spectra combined with the retrieved and quality assessed atomic line data for downloading (see text). 


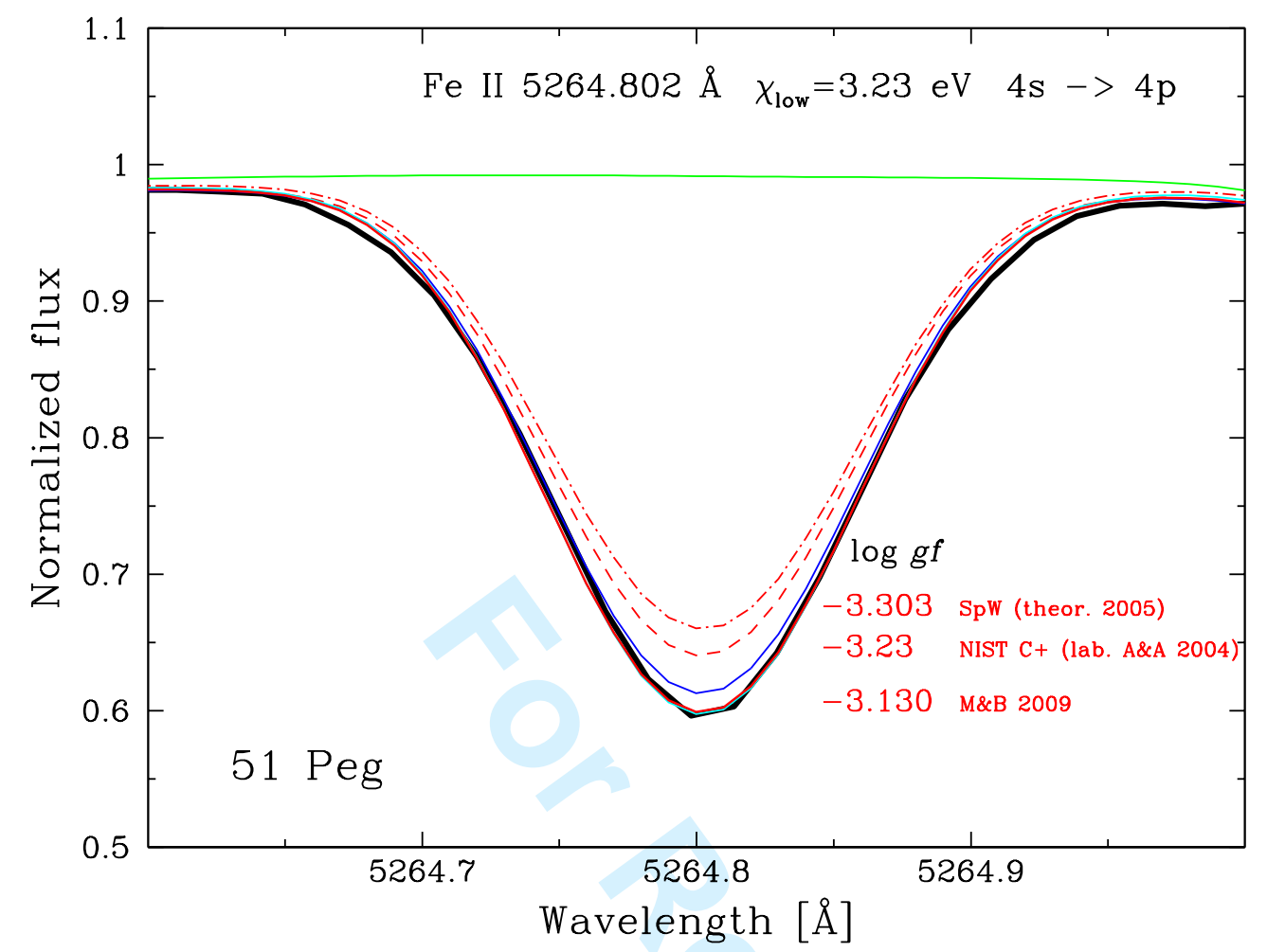

Fig. 5. The Fe II line at $5264.802 \AA(4 \mathrm{~s} \rightarrow 4 \mathrm{p})$ we observe in the BRASS benchmark spectrum of 51 Peg (black solid line) is sufficiently unblended (yellow marked L1 label in Fig. 4) for detailed synthetic spectrum calculations using various $\log (g f)$-values we retrieve from the literature and several atomic line data sources. We find the best fit to the observed line depth for $\mathrm{T}_{\text {eff }}=5804 \mathrm{~K}$ and other APs of Table 1 using $\log (g f)=-3.130$ (solid red line). The blue line is computed for $\mathrm{T}_{\text {eff }}=5674 \mathrm{~K}$. Published $\log (g f)$-values that are considerably smaller do not correctly fit the observed line depth and equivalent width (red broken lines and corresponding marked values). The line fit quality and atomic data accuracy will be assessed using the BRASS benchmark spectra and provided in the BDI (see text). 